\title{
Relación entre algunas técnicas de reparación de hipospadias y complicaciones en el Hospital Universitario San Vicente de Paul 1986- 2005
}

\author{
Martinez Montoya JA*,**, Chams Anturi AA*, Contreras Pérez DF*,**. \\ *Universidad de Antioquia, Hospital San Vicente de Paul**. Medellín, Antioquia. Colombia.
}

Actas Urol Esp. 2008;32(3):332-340

\section{RESUMEN}

RELACIÓN ENTRE ALGUNAS TECNICAS DE REPARACION DE HIPOSPADIAS Y COMPLICACIONES EN EL HOSPITAL UNIVERSITARIO SAN VICENTE DE PAUL 1986- 2005

Objetivo: El éxito en la reparación quirúrgica de hipospadias depende de variables propias del paciente y de variables relacionadas con la técnica quirúrgica. El cirujano dispone de varias técnicas quirúrgicas, lo que obliga ha escoger la técnica mas indicada para obtener los mejores resultados. El objeto de este estudio retrospectivo fue evaluar las técnicas quirúrgicas utilizadas y su asociación con complicaciones postoperatorias.

Metodología: Es un estudio descriptivo y retrospectivo en el cual se revisaron las historia clínicas de 207 pacientes sometidos a corrección de hipospadias en el Hospital Universitario San Vicente de Paúl, entre enero de 1986 y diciembre de 2005.

Resultados: Se revisaron un total de 207 historias clínicas, la edad de cirugía fue entre 1 y 14 años de edad. La frecuencia de las técnicas utilizadas fueron: Duckett 38,7\%, Mathieu 30\%, MAGPI 11.6\%, Snodgrass 6.3\% y otras técnicas representaron en pacientes $14 \%$.

Las complicaciones postoperatorias fueron: Hemorrágicas (1,9\%), Infecciosas (1.9\%), divertículos uretrales $(3,9 \%)$, cuerda ventral residual (5.8\%), dehiscencias $(8,7 \%)$, estenosis $(23,2 \%)$ y fistulas $(41 \%)$.

El desarrollo de estenosis es un factor de riesgo para la aparición de fístulas, y el uso de PDS como material de sutura fue un factor protector estadísticamente significativo.

El cubrimiento de la uretroplastia con otra capa de tejido, la edad para la corrección, y el tiempo que permaneció el paciente con derivación uretral no resultaron variables estadísticamente significativas para la prevención de complicaciones postoperatorias. La estenosis y el uso de PDS como material de sutura si resultaron variables significativas en la reducción de complicaciones postoperatorias. $(\mathrm{p}<0,05)$.

Conclusiones: Algunos factores conocidos como protectores para disminuir la incidencia de complicaciones postoperatorias, como una capa de tejido sobre la línea de sutura, el mayor tiempo de derivación con sonda uretral y la edad temprana del paciente, deben ser evaluadas mas profundamente para determinar su incidencia en la disminución de complicaciones postoperatorias después de la corrección de hipospadias.

Palabras clave: Hipospadias. Técnica quirúrgica. Complicaciones.

\section{ABSTRACT}

RELATION BETWEEN SOME THECNIQUES OF HYPOSPADIAS REPAIR AND COMPLICATIONS: UNIVERSITARY SAN VICENTE DE PAUL HOSPITAL 1986-2005

Objective: The incidence of hypospadias has been increasing in the last decades. Successful hypospadias repair depends on patient factors and surgical technique, a factor closely related to the surgeon's experience. The aim of this descriptive, retrospective study was to determine the preferred technique and its association with postoperative complications.

Methods: Charts of 207 patients who had had hypospadias repair performed between January 1986 and December 2005, in the San Vicente de Paul University Hospital, were reviewed.

Results: Hypospadias repair was performed in patients between 1 and 14 years of age. Duckett technique was performed in 38.7\%, Mathiew technique in 30\% MAGPI technique in $11.6 \%$, Snodgrass in $6.3 \%$ and other techniques in $14 \%$ of the patients. Postoperative complications included hemorrhagic complications (1.9\%), infectious complications (1.9\%), urethral diverticular dilatation (3.9\%), residual ventral chordee (5.8\%), failure of urethroplasty, $(8.7 \%)$, strictures $(23 \%)$, and fistulas $(41 \%)$. Strictures was the most important risk factor for postoperative fistulas ( $>0.05)$. Patients with hypospadias repair with PDS sutures had a lower incidence of postoperative fistulas $(\mathrm{p}<0.05)$. Tissue layer over the suture line, age of patient, and number of days with urethral stent was not related to the presence of postoperative complications.

Conclusion: The importance of known and accepted protective factors in hypospadias repair, like tissue layer over the suture line, age of patient and more or less days with urethral stent, in terms of avoidance of postoperative complications after hypospadias repair, may have to be re-evaluated.

Keywords: Hypospadias. Surgical technique. Complications. 
La incidencia de hipospadias varía de acuerdo a cada región geográfica. El rango de prevalencia va desde 0,26 por cada 1.000 nacimientos en México, hasta 2,1 en Hungría y 2,6 en Escandinavia ${ }^{1}$.

En 1997 el sistema de salud de USA, reportó un aumento del doble de nacimientos de niños con hipospadias, en comparación con décadas anteriores $^{2}$. Además se ha reportado un aumento en la incidencia de hipospadias pasando de 20 por cada 10.000 nacidos en 1970 hasta 40 por cada 10.000 nacidos en $1993^{3}$.

Como agentes etiológicos en esta entidad, se han propuesto entre otros, una producción anormal de andrógenos ${ }^{4}$, una alteración en los receptores androgénicos ${ }^{6}$, alteraciones de la hormona gonadotropina coriónica, además de ingesta de medicamentos durante el embarazo ${ }^{5,8}$.

Existe una incidencia familiar de hipospadias hasta de un 7\%, lo que ha hecho pensar en diferentes componentes genéticos ${ }^{7,31-33}$.

Dentro de las anomalías asociadas a esta entidad, se describe la criptorquidia desde un 10\% hasta un $30 \%$ en hipospadias proximales ${ }^{12}$. Hasta un $15 \%$ de los pacientes con hipospadias pueden presentar hernias inguinales. Raifer reportó que hasta un $27,3 \%$ de pacientes con hipospadias proximal y criptorquidia presentan estados de intersexualidad $^{9,11,30}$. Por otra parte, se ha reportado hasta un $14 \%$ de pacientes hipospádicos con utrículo prostático gigante, lo cual puede ocasionarles infecciones urinarias ${ }^{10}$.

El éxito en la reparación quirúrgica de esta entidad, depende de su severidad. De acuerdo a la localización del meato, se conoce que entre un 50 y $70 \%$ se localiza en el glande (distales), un 20\% a 30\% en el cuerpo del pene (medias), y menos del 10\% a 20\% en la región escrotal o perineal (posteriores) ${ }^{13,14}$.

El cirujano dispone de diferentes técnicas quirúrgicas para lograr un meato ubicado en el ápex del glande, corregir la curvatura ventral del pene, frecuentemente asociada y lograr el mejor resultado estético $^{15}$. Para ello es indispensable escoger la técnica quirúrgica más apropiada para cada paciente, el mejor material de sutura, el tipo de derivación, para obtener el menor número de complicaciones postoperatorias. A pesar de esto, se describen tasas altas de complicaciones postoperatorias que van desde un 5\% hasta un 54\%, siendo las más frecuentes las fístulas, las estenosis y los divertículos ${ }^{16-19}$.
En la Sección de Cirugía y Urología Pediátrica de la Universidad de Antioquia en el Hospital Universitario San Vicente de Paúl de Medellín, se realizan este tipo de correcciones quirúrgicas desde el año 1971, en la actualidad se dispone de una base de datos confiable con la información de los pacientes intervenidos por hipospadias desde 1986.

El presente estudio pretende describir algunas características relacionadas con aspectos demográficos, variables anatómicas, aspectos referentes a la técnica quirúrgica y las complicaciones del procedimiento, en aquellos niños menores de 14 años, con hipospadias corregidas en el Hospital Universitario San Vicente de Paúl entre 1986 y 2005; el análisis de estos resultados permite conocer las características de la población atendida en esta institución, la frecuencias de las diferentes técnicas quirúrgicas, evaluando algunos factores que influyeron en el resultado final. Con el propósito final de establecer las mejores condiciones y técnicas para obtener los mejores resultados.

\section{METODOLOGÍA}

Este trabajo es de tipo descriptivo y retrospectivo, en el cuál se establecen asociaciones de variables. Se tomo el número total de niños con hipospadias intervenidos quirúrgicamente en el Hospital Universitario San Vicente de Paul, entre enero de 1986 y diciembre de 2005.

Las variables estudiadas fueron: Edad, localización del meato, prepucio palmeado, transposición escrotal, grado de retracción ventral del pene, antecedentes gestacionales, patologías asociadas, procedimientos quirúrgicos, material de sutura usado, colgajo de protección para la línea de sutura en la uretroplastia y Derivación, Complicaciones tempranas, tardías y resultado de satisfacción del paciente.

La información fue recolectada mediante la revisión de las historias clínicas de los pacientes, consignando los datos en un formulario con las variables a investigar En los casos en los cuales se considero la información incompleta o insuficiente, se realizo la citación telefónica para consulta del paciente.

Se diseñó una base de datos en el programa SPSS.

En el plan de análisis se describe el comportamiento de las variables estudiadas, se calcularon 
las medias y las desviaciones estándar para las variables cuantitativas, y las frecuencias para las cualitativas. Para establecer asociaciones se calcularon los intervalos RR y OR, uno u otro dependiendo del tipo de estudio.

\section{RESULTADOS}

Entre los años de 1986 y 2005 fueron intervenidos en el Hospital Universitario San Vicente de Paúl un total de 207 pacientes con el diagnóstico de hipospadias.

La edad de los pacientes oscilaba entre el año y los 14 años cumplidos, con una media de 4 años.

Sólo 12 de las madres de los 207 (6\%), admitió haber ingerido algún tipo de medicamento durante el embarazo y en 25 de los 207 pacientes, (12\%) se encontraron antecedentes de hipospadias en algún miembro de la familia.

El número de procedimientos quirúrgicos por año fue variable, presentándose un incremento importante a partir del año 1997 (Fig. 1).

Cuando se agruparon los pacientes estudiados, según la ubicación del meato uretral, en hipospa-

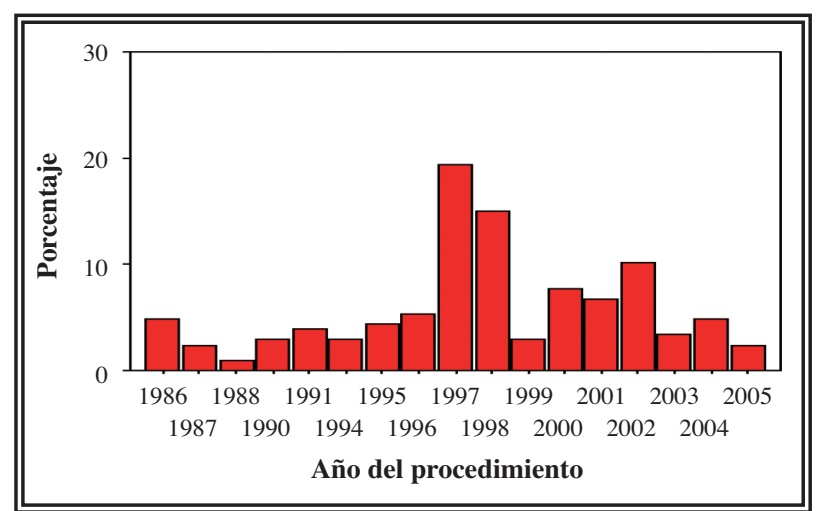

\section{FIGURA 1}

dias proximales, medias y distales, se encontró un $36.7 \%$ de proximales, $26,6 \%$ en la parte media y $36,7 \%$ de distales.

Con relación a las características propias del pene hipospádico encontramos que el $66.7 \%$ de los pacientes estudiados tenían prepucio palmeado, el $18,4 \%$ transposición escrotal y el 58,5\% tenían retracción o cuerda ventral.

Con referencia a otras entidades asociadas con hipospadias, se encontró que el 4,3\% de los pacientes presentaron hernias inguinales asociada a la hipospadias y el 8,2\%, presentaron criptorquidia, siendo unilateral en el $4,8 \%$ de los pacientes y en el resto $(3,4 \%)$ bilateral.

Con respecto al número de procedimientos por paciente, se encontró que en el 76,8\% fue la primera intervención quirúrgica, y en el $23,2 \%$ fueron reintervenciones, así: en el 15\% la segunda, en el $5,8 \%$ la tercera y en el $2,4 \%$ tuvieron 4 o más cirugías. Esto lógicamente afecta los resultados en términos de complicaciones.

De acuerdo a la técnica quirúrgica empleada, durante este tiempo se realizaron 79 Duckett (38,7\%), 62 Mathieu (30\%), 24 MAGPI (11,6\%), 13 Snodgrass $(6,3 \%)$ y otras técnicas representaron en 29 pacientes (14\%).

Con relación a la tasa de complicaciones según la técnica quirúrgica aplicada para la corrección de hipospadias, encontramos lo siguiente:

En el grupo de 24 pacientes en quienes se uso de la técnica MAGPI, no se encontraron complicaciones tempranas, hallándose tasas bajas de complicaciones tardías, dadas por la formación de una fístula (4\%). El resultado estético final en términos de satisfacción para el paciente fue bueno en el 96\% de ellos.

En el grupo de 62 pacientes en quienes se uso de la técnica de MATHIEU se encontraron fístulas en el $17 \%$ y estenosis en el 17\%, obteniéndose resultados estéticos al final de los procedimientos quirúrgicos, descritos como buenos en el 96,7\% de ellos.

Dentro del grupo de 79 pacientes a quienes se les aplicó la técnica de DUCKETT, se encontró una tasa de dehiscencias del 11\%, de fístulas del 58\%, de estenosis del $22 \%$ y de retracción ventral residual del 6\%. Los resultados estéticos fueron considerados como buenos en el $86 \%$ de ellos.

En el grupo de 13 pacientes intervenidos con la técnica SNODGRASS se obtuvo una complicación temprana (infección local) y como complicaciones tardías se presentaron fístulas en el $46 \%$ y estenosis en el 31\% de ellos. Los resultados estéticos fueron considerados como buenos en $84 \%$ de los pacientes.

Cuando se evaluó el material de sutura utilizado en la uretroplastia se encontró que fue Vicryl en el $85 \%$ de los casos y de PDS en el $15 \%$.

En el 48,3\% de los pacientes se realizó protección para la línea de sutura de la uretroplastia con colgajo de tejido vascularizado.

Para la derivación y como tutor uretral, se utilizó la sonda de Nelatón, en el 93,7\% de los pacientes. 
Dentro del grupo global de pacientes se encontró que las complicaciones tempranas fueron definidas como aquellas que se presentaron durante el primer mes de postoperatorio. Se encontraron: dehiscencias $(8,7 \%)$, infecciosas $(1,9 \%)$ y complicaciones hemorrágicas $(1,9 \%)$.

Las complicaciones tardías fueron definidas como aquellas que se presentaron después del primer mes y hasta el primer año de postoperatorio. Entre las complicaciones tardías hubo $41 \%$ de fístulas, $23,2 \%$ de estenosis, $5,8 \%$ de cuerda ventral residual y $3,9 \%$ de divertículos.

Con relación al resultado final percibido por el paciente y consignado en la encuesta de satisfacción, el 91,3\% de lo pacientes consideraron el resultado estético como bueno y el 8,7\% como regular.

\section{Análisis Bivariado Exploratorio}

Al realizar los cruces exploratorios entre variables encontramos lo siguiente:

El 60\% de los pacientes que presentaron estenosis postoperatoria, desarrollaron a su vez fístulas (RR de 1,72 (IC 95\% 1,26-2,34) p=0,0019). Esto confirma que existe una fuerte relación entre estenosis y la posibilidad de desarrollar fístulas.

El 35,4\% de los pacientes sometidos a dos o más procedimientos quirúrgicos, desarrollaron estenosis, en comparación con $19,5 \%$ de los pacientes intervenidos por primera vez. (RR 1,82 (IC 95\% 1,11-2,98) $\mathrm{p}=0$,022). Esto sugiere que la mejor oportunidad para el paciente está en la primera intervención quirúrgica.

Con relación a los materiales de sutura utilizados para la uretroplastia, se encontró que el 43\% de los pacientes en quienes se usó Vicryl, desarrollaron fístula, en contraste con sólo un 19\% de pacientes con fístula, en los que se utilizó el PDS. (RR 2,25 (IC 95\% 1-5,03) $\mathrm{p}=0,02)$. Esto sugiere que el material mas apropiado para la uretroplastia es el PDS.

Cuando se analizó la presencia de estenosis como complicación postoperatoria, con relación a la edad de los pacientes al momento de la uretroplastia, encontramos que en los 96 pacientes menores de 2 años, se presentaron 22 estenosis (23\%) y en los 111 pacientes mayores de 2 años, 26 pacientes presentaron estenosis $(23,4 \%)$. Lo cual no representa una diferencia significativamente estadística. $(p=0,93)$.

Cuando se analizó la presencia de fístulas como complicación postoperatoria, con relación a la edad de los pacientes al momento de la uretroplastia, encontramos que el $48 \%$ de los 96 pacientes menores de 2 años presentaron fístula y en los 111 pacientes mayores de 2 años, el 35\% presentaron fístulas. $\mathrm{p}=0,06$. Por lo tanto cuando se evaluó la edad del paciente, dividiendo el grupo total en mayores y menores de 2 años, con relación a la aparición de fístulas o de estenosis, no se encontró una asociación estadísticamente significativa, dentro del grupo de pacientes analizado en nuestro estudio.

Cuando se analizó el uso de colgajo de tejido vascularizado para protección de la uretroplastia, con relación a la aparición de complicaciones postoperatorias, se encontró que el $21 \%$ de los pacientes en quienes se usó colgajo de protección desarrollaron estenosis, y el 44\% de ellos desarrollaron fístulas, mientras que en el grupo de pacientes en los cuales no se uso ningún colgajo de protección encontramos que un $18 \%$ desarrollaron estenosis $(p=0,56)$ y el $33 \%$ desarrollaron fístula $(\mathrm{p}=0,16)$.

Con relación a los días de uso de sonda de derivación en el postoperatorio, se dividió el grupo total de pacientes en tres grupos, en el primer grupo los pacientes con sonda de cero a cuatro días, en otro grupo los pacientes con sonda entre cinco y diez días y en el ultimo grupo los pacientes con sonda por más de diez días. Cuando se cruzaron estos grupos de pacientes con la aparición de fístulas y estenosis encontramos que en el grupo de cero a cuatro días con sonda, el 40\% presentaron estenosis y el 20\% presentaron fístulas, en el grupo con sonda entre 5 y 10 días, el 23\% presentaron estenosis y el $40 \%$ presentaron fístulas y en el grupo de pacientes que tuvo sonda por mas de 10 días, encontramos que el 19\% de los pacientes tuvieron estenosis y el $47 \%$ desarrollaron fístulas. Sin embargo en ninguna de estas asociaciones se encontró una asociación significativamente estadística (Tablas 1 y 2).

\section{DISCUSIÓN}

Entre el año de 1986 y el 2005 se intervinieron en el Hospital Universitario San Vicente de Paul, un total de 207 pacientes con el diagnóstico de hipospadias. El mayor incremento en el número de procedimientos, ocurre a partir de 1997 y continúa en aumento progresivo aunque en menor cuantía en los años siguientes, especialmente cuando comparamos los primeros 
Tabla 1

\begin{tabular}{|c|c|c|c|c|c|}
\hline \multirow[t]{2}{*}{ Variable } & \multicolumn{2}{|c|}{ Estenosis } & \multirow{2}{*}{$\begin{array}{l}\text { Riesgo } \\
\text { relativo }\end{array}$} & \multirow{2}{*}{$\begin{array}{c}\text { Intervalo } \\
\text { de confianza }\end{array}$} & \multirow{2}{*}{$\begin{array}{l}\text { Valor } \\
\text { de } p\end{array}$} \\
\hline & $\mathbf{S i}$ & No & & & \\
\hline \multicolumn{6}{|l|}{ Edad } \\
\hline Mayor 2 años & 26 & 85 & & & \\
\hline Menor 2 años & 22 & 74 & 1,02 & $0,6-1,6$ & 0,93 \\
\hline \multicolumn{6}{|l|}{ Material de sutura } \\
\hline Vycril & 40 & 136 & & & \\
\hline PDS & 4 & 22 & 1,48 & $0,58-3,79$ & 0,55 \\
\hline \multicolumn{6}{|l|}{ Colgajo } \\
\hline No & 16 & 74 & & & \\
\hline $\mathrm{Si}$ & 17 & 63 & 0,84 & $0,84-1,5$ & 0,56 \\
\hline \multicolumn{6}{|l|}{ Número de Cx } \\
\hline Reintervención & 17 & 31 & & & \\
\hline Primera $\mathrm{Cx}$ & 31 & 128 & 1,82 & $1,11-2,98$ & 0,022 \\
\hline \multicolumn{6}{|c|}{ Número de dias sonda } \\
\hline $0-4$ & 2 & 3 & & & \\
\hline $5-10$ & 30 & 101 & & & \\
\hline$>10$ & 10 & 43 & 0,3 & & 0,34 \\
\hline
\end{tabular}

Tabla 2

\begin{tabular}{|c|c|c|c|c|c|}
\hline \multirow[t]{2}{*}{ Variable } & \multicolumn{2}{|c|}{ Fístula } & \multirow{2}{*}{$\begin{array}{l}\text { Riesgo } \\
\text { relativo }\end{array}$} & \multirow{2}{*}{$\begin{array}{c}\text { Intervalo } \\
\text { de confianza }\end{array}$} & \multirow{2}{*}{$\begin{array}{l}\text { Valor } \\
\text { de } p\end{array}$} \\
\hline & $\mathbf{S i}$ & No & & & \\
\hline \multicolumn{6}{|l|}{ Estenosis } \\
\hline $\mathrm{Si}$ & 29 & 19 & 1,72 & $1,26-2,34$ & 0,0019 \\
\hline No & 56 & 103 & & & \\
\hline \multicolumn{6}{|l|}{ Edad } \\
\hline Mayor 2 años & 39 & 72 & 0,73 & $0,53-1,02$ & 0,06 \\
\hline Menor 2 años & 46 & 50 & & & \\
\hline \multicolumn{6}{|l|}{ Material de sutura } \\
\hline Vycril & 76 & 100 & 2,25 & $1-5,03$ & 0,02 \\
\hline PDS & 5 & 21 & & & \\
\hline \multicolumn{6}{|l|}{ Colgajo } \\
\hline No & 30 & 60 & 0,76 & $0,52-1,12$ & 0,16 \\
\hline $\mathrm{Si}$ & 35 & 45 & & & \\
\hline \multicolumn{6}{|l|}{ Número de $\mathrm{Cx}$} \\
\hline Reintervención & 28 & 23 & 1,38 & $0,99-1,93$ & 0,077 \\
\hline Primera Cx & 60 & 99 & & & \\
\hline \multicolumn{6}{|c|}{ Número de dias sonda } \\
\hline $0-4$ & 1 & 4 & 0,28 & & 0,23 \\
\hline $5-10$ & 53 & 78 & & & \\
\hline$>10$ & 25 & 28 & & & \\
\hline
\end{tabular}

diez años y los últimos diez años. Este incremento sostenido podría atribuirse a primera vista a un aumento en los nacimientos de niños con hipospadias en nuestro medio y también a un mejor posicionamiento del Hospital como centro de referencia de pacientes con hipospadias. Sin embargo, pensamos que el aumento exagerado que se presento en 1997 esta determinado fundamentalmente, por la disponibilidad presupuestal y por las políticas de remisión de las empresas aseguradoras en salud. Una prueba de ello es que el aumento en el número de pacientes, que se presentó en 1997 no se sostuvo igual durante los años siguientes. 
De acuerdo a lo reportado, la criptorquidia se asocia a hipospadias en un 8-9\% y a la hernia inguinal y el hidrocele en un 9 al 16\%10,68,71. En este estudio encontramos que el $4,3 \%$ de los pacientes presentaron hernias inguinales asociada a la hipospadias y el $8,2 \%$, presentaron criptorquidia, siendo unilateral en el $4,8 \%$ de los pacientes y en el resto $(3,4 \%)$ bilateral.

Con relación a la distribución de los tipos de hipospadias de acuerdo con la ubicación del meato uretral, sabemos que en la literatura existe una mayor proporción de niños con hipospadias distal (50\% a 70\%), un porcentaje intermedio para pacientes con hipospadias media (30\%) y el menor porcentaje para los pacientes con hipospadias proximal $(20 \%)^{1}$. Sin embargo, en nuestro estudio se presentaron porcentajes diferentes, así: con hipospadias distal el 37\% de los pacientes, con hipospadias media el $27 \%$ y con hipospadias proximal el 37\%. Esto puede indicar que existe una mayor tendencia a intervenir a los pacientes con hipospadias distales, en otros centros quirúrgicos, por ser cirugías, teóricamente mas sencillas y con menor riesgo de complicaciones, esto explicaría un porcentaje menor en este grupo de pacientes $(37 \%)$, por otro lado, con respecto a las hipospadias proximales encontramos un mayor número de pacientes (37\%) respecto a lo reportado en la literatura (20\%), esto puede indicar una mayor tendencia a remitir los pacientes con hipospadias proximales por ser correcciones quirúrgicas mas complejas y con mayor riesgo de complicaciones. Este dato si podría sugerir que existe un mejor posicionamiento del Hospital Universitario San Vicente de Paúl como centro de referencia de patología urológica compleja en los pacientes pediátricos.

Con relación a las características del pene hipospádico, encontramos que el $67 \%$ de los pacientes tenían prepucio palmeado, el 59\% una retracción ventral del pene y el 18\% algún grado de transposición escrotal. Estos porcentajes están determinados por la frecuencia de pacientes con los diferentes grados de hipospadias y se relacionan con los porcentajes informados en otros estudios similares.

Con relación a las complicaciones tempranas, identificadas como hemorragia, infección o dehiscencia de la sutura, en este estudio fueron mínimas, en relación a lo descrito en la literatura.

El numero de complicaciones tardías encontrado, fístulas (38\%) y estenosis (19\%), corresponden a lo reportado en otras series aun cuando se han llegado a reportar en la literatura de hasta un 50\% de fístulas.

Cuando comparamos los resultados de los primeros diez años del estudio con los diez años últimos vemos una disminución de las complicaciones tardías, lo cual puede atribuirse a una mayor experiencia y una técnica quirúrgica más depurada, atribuible a la curva de aprendizaje normal.

El hecho de haber tenido un alto número de pacientes remitidos para reintervención o con hipospadias más proximales que distales, modifica desfavorablemente nuestros resultados postoperatorios en relación con fístulas y estenosis.

Cuando se cruzaron las variables estenosis y fístulas se encontró que aquellos pacientes que presentaron estenosis tuvieron 1,8 veces mas probabilidad de desarrollar fístula, lo cual es estadísticamente significativo. Esto lógicamente, se explica por la mayor presión ejercida por la orina en la uretra ante un fenómeno obstructivo distal.

Con relación a la edad óptima para la cirugía, se acepta que teniendo en cuenta: el conocimiento de las implicaciones fisiológicas de la cirugía genital en niños, los mejores desarrollos técnicos para la cirugía y la anestesia pediátrica, la mejor edad para la reparación quirúrgica de hipospadias, está entre los 6 y los 12 meses de edad $^{47-49}$. De tal manera que la reparación de hipospadias antes del primer año de edad se ha convertido en una conducta generalizada actualmente. Otros estudios sugieren también, que existe una tasa significativamente mayor de complicaciones postoperatorias en pacientes mayores, y recomiendan la reparación en edades tempranas ${ }^{86}$. Sin embargo, en el presente estudio, la edad del paciente no pudo relacionarse en forma estadísticamente significativa cuando se comparó con el desarrollo de fístulas o de estenosis.

Teniendo en cuenta que un $25 \%$ de las cirugías fueron reintervenciones en pacientes remitidos después de una primera intervención fallida, este estudio determinó que los paciente reintervenidos tienen dos veces mas probabilidad de desarrollar estenosis y fístulas que los operados por vez primera, claramente entre más intervenciones quirúrgicas más se altera la cantidad y la calidad de los tejidos para la reconstrucción. 
Con relación a los detalles de la técnica quirúrgica y a los materiales utilizados, se acepta que el aspecto técnico más importante es la superposición exacta de los bordes de los tejidos en la uretroplastia, logrando que la superficie epitelializada quede invertida, en su totalidad y la superficie cruenta de tejido subepitelial quede bien aproximado. En estas condiciones el proceso de cicatrización asegura una línea de sutura a prueba de agua, lo cual disminuye el riesgo de fístulas. Sin embargo Hakim en 1996, informa en su estudio que la técnica de sutura ya sea subcuticular o sutura continua de espesor total no afecta el resultado final, usando una sutura multifilamento absorbible de tipo Poligalactina ${ }^{85}$. Por el contrario Ulman en $1997^{87}$ y Disandro y Palmer en $1996^{84}$ encontraron una menor incidencia de fístulas utilizando PDS que Poligalactina (Vicryl).

En nuestro estudio encontramos, en los niños en quienes se utilizo PDS como material de sutura para la uretroplastia, tuvieron 2,25 veces menos posibilidad de desarrollar fístulas y 1,5 veces menos de desarrollar estenosis, que cuando se utilizó Poligalactina (Vicryl).

Inexplicablemente, en este estudio, a diferencia de estudios previos ${ }^{75,76}$, la presencia de un colgajo de tejido para protección de la línea de sutura en la uretroplastia, no pudo relacionarse en forma significativamente estadística con una menor incidencia de fístulas o de estenosis. Para la mayoría de los autores este es un factor protector determinante para evitar las fístulas, sin embargo, falta estudio para determinar si en algunos casos el uso de un colgajo de tejido para cubrir la anastomosis, puede aumentar el riesgo de estenosis.

El número de días que el paciente permaneció con sonda uretral postoperatoria, a pesar de haber clasificado los pacientes de acuerdo a la técnica quirúrgica, no pudo relacionarse en forma estadísticamente significativa con las complicaciones tardías como fístulas y estenosis. Posiblemente, el tiempo con sonda uretral postoperatoria no constituya un factor determinante en la aparición de complicaciones, es decir, podría ser lo mismo dejar la sonda por tres días a dejarla por diez días. La aclaración de este interrogante debe ser motivo de estudios adicionales dentro de esta línea de investigación.

\section{CONCLUSIONES}

1. Como ocurre a nivel mundial, la incidencia de hipospadias en nuestro medio ha aumentado progresivamente en los últimos 10 años. Sin embargo, los presupuestos y las políticas de remisión de los pacientes por parte de las empresas promotoras de salud, puede modificar en forma importante el número de pacientes intervenidos en un solo año.

2. La asociación de criptorquidia y hernias inguinales con hipospadias, encontrados por nosotros, se asemeja a los porcentajes informados por otros autores.

3. La asociación de prepucio palmeado, cuerda ventral y transposición escrotal obedece mas al tipo de hipospadias de los pacientes intervenidos que a una muestra representativa de la población general.

4. El $64 \%$ de los pacientes intervenidos correspondió a niños con hipospadias proximales (37\%) y medias (27\%). Por otro lado, el 25\% de ellos fueron reintervenciones. Esto modificó desfavorablemente el resultado final en términos de aparición de complicaciones como fístulas (38\%) y estenosis (19\%).

5. El alto riesgo de complicaciones postoperatorias en los niños con hipospadias obliga a que sean tratados por personal altamente entrenado y en un centro de referencia para este tipo de cirugías reconstructivas, siendo el primer procedimiento la mejor oportunidad para el paciente.

6. El desarrollo de estenosis como complicación postoperatoria es un factor de riesgo evidente para el desarrollo de fístulas.

7. La utilización de PDS en la uretroplastia, mostró menor número de complicaciones, respecto al uso de poliglactina (Vicryl).

8. La menor edad al momento de la cirugía, no disminuye por sí misma, el riesgo de desarrollar complicaciones.

9. El cubrimiento de la línea de sutura en la uretroplastia con un colgajo de tejido vascularizado, no disminuyó el riesgo de complicaciones.

10. El número de días de derivación con sonda uretral, para cada técnica quirúrgica, no resultó ser un factor significativo para determinar el desarrollo de complicaciones.

\section{REFERENCIAS}

1. Duckett JW: Hypospadias .In Walsh PC, Retik AB, Vaughan ED, Jr, Wein AJ, (Eds): Campbell`s Urology, vol 2. Philadelphia, WB Saunders, 1998, pp 2093-2119.

2. Paulozzi LJ, Ericsson JD, Jackson RJ. Hipospadias trends in two US surveillance systems. Pediatrics. 1997;100(5):831-834. 
3. Dolk H. Rise in prevalence of hypospadias. Lancet 1998;351 (9105):770.

4. Aaronson IA, Cakmark MA, Key LL. Defects on the testosterona biosinthetic pathway in boys with hypospadias. J Urol. 1997;157(5):1884-1888.

5. Aarskog D. Maternal progestins as a possible cause of hypospadias. N Engl J Med. 1979;300(2):75-78.

6. Allen TD, Griffin JE. Endocrine studies in patients with advanced hypospadias. J Urol. 1984;131(2):310-314.

7. Bassin LS, Liu W, Li Y, et al: FGF-10 gene disruption results in hypospadias. In section on Urology Program for Scientific sessions. 2000 Annual Meeting of the American Academy of Pediatrics. Oct 282000.

8. Mau G: Progestins during pregnancy and hypospadias. Teratology. 1981;24(3):285-287.

9. Kaefer M, Diamond D, Hendren WH, Vemulapalli S, Bauer SB, Peters CA, et al. The incidence of intersexuality in children with cryptorchidism and hypospadias. J Urol. 1999:162(3Pt2): 1003-1006.

10. Khuri FJ, Hardy BE, Churchill BM. Urologic anomalies associated with hypospadias. Urol Clin North Am. 1981;8(3):565-571.

11. Rajfer J, Walsh PC. The incidence of intersexuality in patients with hypospadias and cryptorchidism. J Urol. 1976;116(6): 769-770.

12. Yamaguchi T, Kitada S, Osada Y. Chromosomal anomalies in cryptorchidism and hypospadias. Urol Int. 1991;47(2):60-63.

13. Duckett JW: Foreword: Symposium on hypospadias. Urol Clin North Am 1981;8(3):371-373.

14. Barcat J. Current concepts of treatment. Boston Little, Brown, 1973.

15. Mouriquand PD, Persad R, Shama S. Hypospadias repair: current principles and procedures. Br J Urol. 1995;76 Suppl 3:922 .

16. Duckett JW Jr, Kaplan GW, Woodard JR, Devine CJ Jr. Panel: Complications of hypospadias repair. Urol Clin North Am. 1980; 7(2):443-454.

17. Zaontz MR, Kaplan WE, Maizels M. Surgical correction of anterior urethral diverticula after hypospadias repair in children. Urology. 1989;33(1):40-42.

18. Horton CE, Devine CJ Jr. A one-stage repair for hypospadias cripples. Plast Reconstr Surg. 1970;45(5):425-430.

19. Retik AB, Keating M, Mandell J. Complications of hypospadias repair. Urol Clin North Am. 1988;15(2):223-236.

20. Cendron J, Melin Y. Congenital curvature of the penis without hypospadias. Urol Cin North Am. 1981;8(3):389-395.

21. Devine JC Jr, Horton CE. Chordee without hypospadias. J Urol. 1973;110(2):264-271.

22. Glenister JW. The origin and fate of the urethral plate in man. J Anat. 1954;88(3):413-418.

23. Kursrock EA, Baskin LS, Cunha GR. Ontogeny of de male urethra: Theory of endodermal differentiation. 1999, pp 80-90.

24. Stephens FD, Smith ED, Hutson JM: Embryogenesis of hypospadias. In Stephens FD (ed): Congenital Anomalies of the urinary and genital tracts. Oxford, UK: Isis Medical Media Ltd, 1996, pp 80-90.

25. Snodgrass W, Patterson K, Plaire JC, Grady R, Mitchell ME. Histology of the urethral plate: Implications for hypospadias repair. J Urol. 2000;164(3 Pt 2):988-990.

26. Marshall M Jr, Beh WP, Johnson SH 3rd, Price SE Jr, Barnhouse DH. Etiologic considerations in penoescrotal hypospadias repair. J Urol. 1978;120(2):229-231

27. Donahoo KK, Cain MP Pope JC. Etiology, management and surgical complications of congenital Chordee witout hypospadias. J Urol. 1998;160:1120-1122.
28. Baskin LS, Erol A, Li YW. Anatomical studies of hypospadias. J Urol. 1998; 160(3 Pt 2): 1108-1115. Discussion 1137.

29. Hunter RH. Notes on the development of the prepuce. J Anat. 1935;70(Pt 1):68-75.

30. Aarskog D. Intersex conditions masquerading a simple hypospadias. Birth Defects Original article Series. 1971;7(6): 122-130.

31. Bauer SB, Retick AB Colodny AH. Genetic aspects of hypospadias. Urol Clin North Am. 1981;8(3):559-564.

32. Bauer SB, Bull MJ, Retik A. Hypospadias a familial study. J Urol. 1979;121(4):474-477.

33. Roberts CJ, Lloyd S. Observations on the epidemiology of simple hypospadias. Br Med J. 1973;1(5856):768-770.

34. Sorensen HR. Hypospadias with especial reference to aetiology. Copenhagen, Ejnar Munksgard, 1953.

35. Culp OS. Experiences with 200 hypospadyacs: Evolution of a therapeutic plan. Surg Clin North Am. 1959;39(4):1007-1023.

36. Hatch DA, Maizels M, Zaontz MR, Firlit CF. Hypospadias hidden by a complete prepuce. Surg Gynecol Obstet. 1989;169(3): 233-234.

37. Cerasaro TS, Brock WA, Kaplan GW. Upper urinary tract anomalies associated with congenital hypospadias: Is screening necessary? J Urol. 1986;135(3):537-538.

38. Davenport M, MacKinnon AE. The value of ultrasound screening of the upper urinary tract in hypospadias. Br J Urol. 1988; 62(6):595-596.

39. Mcardle F, Lebowitz R. Uncomplicated hypospadias and anomalies of upper urinary tract: Need for screening. Urology. 1975; 5(5):712-716.

40. Smith EP, Wacksman J. Evaluation of severe hypospadias. J Ped Surg. 1997;131(3):344-346.

41. Gerhart JP, Jeffs RD. The use of parenteral testosterone therapy in genital reconstructive surgery. J Urol. 1987;138(4 Pt 2):10771078.

42. Koff SA, Jaiyanthi VR. Preoperative treatment with human chorionic gonadotropin in infancy decreases the severity of proximal hypospadias and chordee. J Urol. 1999;162(4):1435-1439.

43. Mc Mahon DR, Kramer SA, Husmann DA. Micropenis: Does early treatment with testosterone do more harm than good? J Urol. 1995; 154(2 Pt 2):825-829.

44. Monfort G, Lucas C. Dihydrotestosterone penile simulation in hypospadias surgey. Eur Urol. 1982;8(4):201-203.

45. Paulozzi LJ. Is hypospadias an environmental birth defect .The impact of eviromental and endocrine disruptors in pediatric urology. Dial Pediatr Urol. 2000;23:8.

46. Duckett JW: The current hype in hypospadiology. Br J Urol. 1995;76 suppl 3:1-7.

47. Kass E, Kogan SJ, Manley C. Timing of elective surgery on the genitalia of male children with particular reference to the risk, benefits and psychological effects of surgery and anesthesia. Pediatrics. 1996;97:590-594.

48. Kelalis P, Bunge R, Baskin M. The timing of elective surgery on the genitalia of male children with particular reference to undescended testes and hypospadias. Pediatrics. 1975;56:479-483.

49. Schultz JR, Klykylo WM, Wacksman J. Timing of elective hypospadias repair in children Pediatrics. 1983; 71:342-351.

50. Smith ED: The history of hypospadias. Pediatr Surg Int. 1997; 12:81-85.

51. Borer JG, Retik AB. Current trends in hypospadias repair. Urol Clin North Am. 1999;26:15-37.

52. Rabinowits R. Outpatient catheter less, modified Mathieu hypospadias repair. J Urol. 1987;138:1074-1076.

53. Duckett JW. MAGPI(Meatoplasty and glanduloplasty): A procedure for subcoronal hypospadias. Urol Clin North Am. 1981; 8:513-519. 
54. Mathieu P. Traitement en un temps de I'hypospadias balanique ou juxtabalanique. Journal de Chirurgie 1932;39:481-486.

55. Snodgrass W. Tabularized, incised plate urethoplasty for distal hypospadias. J Urol 1994;151:464-465.

56. Elder JS, Duckett JW, Snyder HM. Onlay island flap on the repair of mid and distal penile hypospadias without chordee. J Urol. 1987; 138:376-379.

57. Duckett JW: Transverse preputial island flap technique for repair of severe hypospadias. Urol Clin North Am. 1980;7:423430.

58. Snodgrass W, Koyle M, Manzoni G et al. Tabularized incised plate hypospadias repair for proximal hypospadias. J Urol. 1998;159:2129-2131.

59. Duckett JW, Coplen D, Ewalt D et al. Buccal mucosal urethral replacement. J Urol. 1995; 153:1660-1663.

60. Memmelaar J. Use of bladder mucosa in a one stage repair of hypospadias. J Urol. 1947;58:68

61. Nesbit R: Congenital curvature of the phallus: Report of three cases with description of corrective operation. J Urol. 1965;93: 230-232.

62. Horton CE Jr, Horton CE. Complications of hypospadias surgery. Clin Plast Surg. 1988;15:371-379.

63. Duckett JW, Kaplan GW, Woodward JR et al. Panel: Complications of hypospadias repair. Urol. Clin North Am. 1980;7 433-454.

64. Keating MA, Duckett JW. Failed hypospadias repair: In Cohen MS, Resnick MI (eds): Reoperative urology. Boston, Little, Brown, 1995, pp 187-204.

65. Kumar MV, Harris DL. Balanitis Xerotica Obliterans complicating hypospadias repair. Br J Plast Surg. 1999;52:69-71.

66. Baskin LS, Duckett JW. Mucosal grafts in hypospadias and stricture management. AUA .Update Series. 1994;13:270275

67. Winslow BH, Devine CJ Jr. Principles in repair of hipospadias. Semin Pediatr Surg. 1996;5:41-48.

68. Sorber M, Feitz WF, de Vires JD. Short and mid-term outcome of different types of on stage hypospadias corrections. Eur Urol. 1997;32:475-479.

69. De Sy Wa. Aesthetic repair of meatal stricture. J Urol. 1984; 132:678-679.

70. Horton CE, Devine CJ Jr. A one stage repair for hypospadias cripples. Plast reconstr Surg. 1970;45:425-430.

71. Sweet RA, Schrott HG, Kurland R et al. Study of the incidence of hypospadias in Rochester, Minnesota, 1940-1970 and a case control comparison of possible etiologic factors. Mayo Clin Proc. $1974 ; 49: 52-58$
72. Nesbit RM. Plastic procedure for correction of hypospadias. J Urol. 1941;45:699-702.

73. Byars LT. Technique of consistently satisfactory repair of hypospadias. Surg Gynecol Obstet. 1955; 100:184-190.

74. Zaontz MR. The GAP (glans approximation procedure) for granular/coronal hypospadias. J Urol 1989; 141:359-361.

75. Belman AB. Hypospadias update. Urology 1997; 49: 166-172.

76. Smith D. A de-epithelialised overlap flap technique in the repair of hypospadias. Br J Plast Surg. 1973; 26:106-114.

77. Baskin LS, Duckett JW. Dorsal tunica albuginea plication for hypospadias curvature. J Urol. 1994; 151:1668-1671.

78. Borer JG, Bauer SB, Peters CA et al. Tubularized, incised plate urethroplasty: Expanded use in primary and repeat surgery for hypospadias. J Urol. 2001;165:581-585.

79. Hinman F Jr. The blood supply to preputial island flaps. J Urol $1991 ; 145: 1232-1235$.

80. Horton CE, Devine CJ Jr. Simulated erection of the penis with saline injection, a diagnostic maneuver. Plast Reconstr Surg. 1977;59:138-139.

81. Joseph DB, Perez LM: Tunica vaginalis onlay uretrhoplasty as a salvage repair. J Urol. 1999;162:1146-1147.

82. Baskin LS, Duckett JW, Ueoka K et al. Changing concepts of hypospadias curvature lead to more onlay island flaps procedures. J Urol. 1994;151:191-196.

83. Rushton HG, Belman AB. The split prepuce in situ onlay hypospadias repair. J Urol 1998; 160:1134-1136; discussion, 1137

84. Disandro M, Palmer JM. Stricture incidence related to suture material in hypospadias surgery. J Ped Surg. 1996;31:881-884.

85. Hakim S, Merguerian PA, Rabinowitz R et al. Outcome analysis of the modified Mathieu hypospadias repair: Comparison of stented and unstented repairs. J Urol. 1996;156:836-838.

86. Hensle T, Tennenbaum S, Reiley E et al. Hypospadias repair in the adult population: Adventures and misadventures. J Urol. 2001;165:77-79.

87. Ulman I, Erikci V, Avanoglu A et al. The effect of suturing technique and material on complication rate following hypospadias repair. Eur J Pediatr Surg. 1997;7:156-157.

Correspondencia autor: Dr. J.A. Martínez Montoya

Hospital Universitario San Vicente de Paul

Medellín, Colombia.

E-mail autor: cxpediatrica@medicina.udea.edu.co

Información artículo: Original - Urología Pediátrica - Hipospadia

Trabajo recibido: junio 2006

Trabajo aceptado: noviembre 2007 